\title{
INPUT TESTING IN THE DETECTION OF MISSPELLINGS
}

\author{
Donald G. MacKay \\ University of California at Los Angeles
}

Two types of misspellings (phonetically compatible, wERK for wORK; or phonetically incompatible, WARK for wORK) were tachistoscopically presented to 24 subjects. Telling them what word would be misspelled increased the probability of detecting phonetically incompatible misspellings, but not phonetically compatible ones. The findings present difficulties for passive models of perception; an active, input-testing model is more promising.

It may seem surprising that spelling errors should provide a testing ground for general issues in perception. But current data on detection of misspellings contradict the general assumption that perception results from direct or passive activation of analyzers for orthographic input. Rather, an active or mismatch process seems to be involved, whereby perception results from a comparison of the input (at various stages of coding) with a self-generated model of that input.

Consider these two types of misspellings: phonetically compatible and phonetically incompatible. Compatible misspellings can be pronounced the same as the original word (say, vURSE for VERSE) whereas the incompatible ones cannot (VORSE for VERSE). MacKay (1968) showed that incompatible misspellings were easier to detect in a sentence than compatible ones, a finding in accord with a mismatch model of perception (after Teuber, 1960). According to this model, the context of the sentence leads the subject to expect a particular word at the phonetic level, and detecting the misspellings depends in part on a mismatch between this expectation and the internal pronunciation of the actual input. Since the internal pronunciation of phonetically compatible misspellings matches that of the expected word, these misspellings pass undetected at the phonetic level. But the expectation fails to match the internal pronunciation of phonetically incompatible misspellings, which are therefore easily detected at the phonetic level according to the mismatch model.

The present study was a replication and extension of the 1968 MacKay study. To control for exposure duration, the phonetically compat- 
ible and incompatible misspellings were presented tachistoscopically. The subject's expectations were controlled by instructing or not instructing him to expect a particular word. According to the mismatch model, phonetically incompatible misspellings should be easier to detect in the instructed condition than in the uninstructed condition. And phonetically compatible misspellings should be harder to detect than incompatible ones in the instructed condition but not in the uninstructed condition.

We also presented misspellings that form new words; for example, the misspelling of GREET as GREAT. Our question was whether subjects would identify GREAT more readily if they expected GREET than if they had no expectation at all. The mismatch model predicts that these 'new word' misspellings should behave like phonetically incompatible ones, being easy to detect in the instructed condition because of an internal mismatch at the pronunciation level and perhaps at the semantic level as well. We will see that the failure of this prediction leads to a theoretical reformulation of the mechanisms underlying the detection of misspellings.

\section{METHOD}

- Subjects and procedure-Twenty-four UCLA undergraduates received course credit for their participation in the 40-minute experiment. Each subject was warned that the words to be presented would sometimes be correctly spelled and sometimes not. He was to write down exactly what he saw, guessing at the spelling if necessary.

The materials consisted of 14 correctly spelled words (say, INCITE), 14 phonetically compatible misspellings (INSITE), and 14 phonetically incompatible misspellings (INBITE). The stimuli were typed in capital letters on 46 5-by-4in. cards, which were shuffled thoroughly for each subject. The two types of misspelling were formed by changing exactly the same letter in one of the correctly spelled words. The misspellings altered neither word length nor letter height. The first letter was always correct but the position of the substituted letter varied from word to word. In addition, 5 new-word misspellings were formed by changing exactly the same letter in the original, correctly spelled words (say, INviTE for INCITE). The new-word misspellings slightly exceeded the original words in average Thorndike-Lorge frequency, but this is irrelevant here, since we were interested in comparing each word with itself in the 'set' and 'no-set' conditions described below.

In the no-set condition, a warning tone sounded $4 \mathrm{sec}$ before the stimulus appeared. The set condition was identical except that the subjects were verbally told what word to expect in the $4 \mathrm{sec}$ between the tone and stimulus onset. The order of the set and no-set conditions was counterbalanced across subjects. Half the stimuli were presented in the set condition and the remainder 
in the no-set condition (counterbalanced across subjects so that each stimulus appeared equally often in both conditions). In both conditions all the materials were first exposed for $120 \mathrm{msec}$ and then for $160 \mathrm{msec}$.

-Apparatus-A two-channel tachistoscope (Scientific Prototype 800F) presented the materials. One channel displayed a fixation point, and the other exosed the stimuli for either 120 or $160 \mathrm{msec}$. The words were $14 \mathrm{~min}$ (visual angle) in height and $30.5 \mathrm{~min}$ to $61 \mathrm{~min}$ in length. The luminance level of the stimuli was about $78 \mathrm{ftL}$ as measured with a Spectra brightness spot meter. The tachistoscope was connected in series to three general purpose timers: one triggered the stimulus onset $4 \mathrm{sec}$ after a warning tone, another fixed the duration of the warning tone $(1 \mathrm{sec})$, and the third began the next trial by triggering the warning tone $12 \mathrm{sec}$ after offset of the stimulus. An HP audio oscillator (201C) generated the $2,000-\mathrm{Hz}$ warning tone.

\section{RESULTS}

\section{Detection of misspellings}

Compatible misspellings were no harder to detect than incompatible ones in the no-set condition. Here the probability of detecting compatible misspellings (averaged across subjects, words, and exposure durations) was .27; incompatible misspellings, .26. Nor did the set condition have a significant effect on the recognition of compatible misspellings ( $p$ at the .50 level, Friedman two-way analysis of variance on ranks). But the set condition did facilitate detection of incompatible misspellings. Significantly more incompatible misspellings were detected in the set than no-set condition $(p<.05$, same test). Similarly, incompatible misspellings were significantly easier to detect than compatible ones in the set condition $(p<.05$, same test).

We categorized incorrect responses as incorrect-word responses and as incorrect-set responses. An incorrect-set response was scored whenever the subject erroneously responded with the 'set' word, and an incorrectword response whenever he erroneously responded with a correctly spelled English word. Table 1 shows the frequency of incorrect-set and -word responses for compatible and incompatible misspellings. As can be seen there, incorrect-word responses were no more frequent for compatible than incompatible misspellings in the no-set condition, but much more frequent in the set condition $(p<.05$, Friedman two-way analysis of variance on ranks). This difference was only partly due to the slightly higher frequency of incorrect-set responses for phonetically compatible misspellings. In the set condition (also shown in Table 1), incorrectword responses remained about $8 \%$ higher for compatible than incompatible misspellings when the incorrect-set responses were subtracted 
Table 1. The probability of incorrect-word responses and incorrect-set responses given an incorrect identification of phonetically compatible and incompatible misspellings

\begin{tabular}{lcccccc}
\hline & \multicolumn{2}{c}{ Incorrect-word responses } & & \multicolumn{2}{c}{ Incorrect-set responses } \\
\cline { 2 - 3 } \cline { 5 - 6 } & $\begin{array}{c}\text { Compatible } \\
\text { misspellings }\end{array}$ & $\begin{array}{c}\text { Incompatible } \\
\text { misspellings }\end{array}$ & & $\begin{array}{c}\text { Compatible } \\
\text { misspellings }\end{array}$ & $\begin{array}{c}\text { Incompatible } \\
\text { misspellings }\end{array}$ \\
\hline Set condition & .71 & .56 & & .40 & .33 \\
No-set condition & .54 & .53 & & .10 & .09 \\
\hline
\end{tabular}

out. Thus compatible misspellings were mistaken for correctly spelled words more often than incompatible misspellings, but only in the set condition.

\section{Detection of correctly spelled words}

'Set' words (say, HERD) and new-word misspellings (say, HARD for HERD) made up the correctly spelled words. The set words were correctly identified more frequently in the set than the no-set condition $(p<.01$, Friedman two-way analysis of variance on ranks). But the opposite was true of new-word misspellings, which were correctly recognized significantly more often in the no-set than in the set condition $(p<.05$, same test).

Incorrect-set responses and incorrect-word responses were scored as before, with the results shown in Table 2. Incorrect-word responses were no more frequent for new-word misspellings than set words in the no-set condition, but were significantly more frequent for new-word misspellings in the set condition ( $84 \%$ versus $0 \%$ ). This difference is only partly due to the high frequency of incorrect-set responses for new-word misspellings (also shown in Table 2). With these incorrect-set responses subtracted out, $50 \%$ of the erroneous responses to new-word misspellings were incorrect-word responses (as compared to 0\% for the set words).

Table 2. The probability of incorrect-word responses given an incorrect response to two types of normally spelled words, along with the probability of incorrect-set responses for new-word misspellings

\begin{tabular}{lcccc}
\hline & \multicolumn{2}{l}{ Incorrect-word responses } & & Incorrect-set responses \\
\cline { 2 - 3 } & $\begin{array}{c}\text { Set } \\
\text { words }\end{array}$ & $\begin{array}{c}\text { New-word } \\
\text { misspellings }\end{array}$ & & $\begin{array}{c}\text { New-word } \\
\text { misspellings }\end{array}$ \\
\hline Set condition & .0 & .84 & .34 \\
No-set condition & .40 & .41 & .08 \\
\hline
\end{tabular}


Apparently the subjects realized the new-word misspellings were words, but were unsure which words. ${ }^{1}$

\section{DISCUSSION}

Our findings present difficulties for passive models of perception. But the mismatch model developed in the introduction also fails to explain certain aspects of our data. According to this model, new-word misspellings should be easier to detect in the set than the no-set condition. Since the reverse was true, we were forced to develop a new model to explain our results. Our model can be considered an input-testing theory (after MacKay, 1967; Miller, Galanter, and Pribram, 1960), since perception is considered to follow a series of tests on the input.

Four possible input tests in the set condition of our experiment are shown in Table 3. Exposure duration was undoubtedly too brief to allow complete application of the first test (Is the input identical to the set word at the orthographic level?). And assuming that only one or two of the remaining tests can be applied on a probabilistic basis, this model seems capable of handling virtually all of our results.

Consider the possibility that a new-word misspelling has only passed test 3 (Is the input identical to the set word at the phonetic level?), with-

Table 3. The outcomes of four possible input tests in the set condition for the four types of stimuli

\begin{tabular}{lcccc}
\hline \multicolumn{1}{c}{ Input tests } & $\begin{array}{c}\text { Phonetically } \\
\text { compatible } \\
\text { misspellings }\end{array}$ & $\begin{array}{c}\text { Phonetically } \\
\text { incompatible } \\
\text { misspellings }\end{array}$ & $\begin{array}{c}\text { Set } \\
\text { words }\end{array}$ & $\begin{array}{c}\text { New-word } \\
\text { misspellings }\end{array}$ \\
\hline $\begin{array}{l}\text { 1. Is the input identical } \\
\text { to the set word at the } \\
\text { orthographic level? }\end{array}$ & No & No & Yes & No \\
$\begin{array}{l}\text { 2. Is the input a familiar } \\
\text { configuration (is it a } \\
\text { word) at the ortho- } \\
\text { graphic level? }\end{array}$ & No & No & Yes & Yes \\
$\begin{array}{l}\text { 3. Is the input identical } \\
\text { to the set word at the } \\
\text { phonetic level? }\end{array}$ & Yes & No & Yes & No \\
$\begin{array}{l}\text { 4s the input familiar } \\
\text { (is it a word) at the } \\
\text { phonetic level? }\end{array}$ & Yes & No & Yes & Yes \\
\hline
\end{tabular}


out time for further tests. Given no further information, the subject's best response is that the set word was presented, which accounts for both the high frequency of incorrect-set responses and the low probability of detecting new-word misspellings in the set condition. Note too that phonetically compatible misspellings pass tests 3 and 4 , making the set word the best possible response if time permits no further tests. The input-testing model therefore explains the high frequency of incorrectset responses for phonetically compatible misspellings.

The model also explains the ease of detecting phonetically incompatible misspellings, which fail all four input tests. The high frequency of incorrect-word responses for phonetically compatible misspellings and new-word misspellings can be handled in similar fashion. Both classes of stimuli pass test 4 (Is the input a word at the phonetic level?), forcing the subject to respond with a word if no further tests can be run. Finally, the model explains why erroneous responses for set words in the set condition were usually phonetically compatible. If the exposure duration permits all but test 1 (Is the input identical to the expected word at the orthographic level?), only two responses are feasible in this condition: one a possible incorrect-set response, and the other a phonetically compatible response. By chance alone, some of the responses will fall into this latter class, explaining the tendency to make errors that are phonetically compatible with the set word.

An input-testing model also captures the difficulty in detecting the absence of silent E's, as in HORDE, or silent K's, as in KNIGHT (Corcoran, $1966,1967,1968)$, since the missing element is absent both in the phonetic test and in the internal pronunciation of the misspelling. The model likewise predicts difficulty in detecting the addition of silent letters as in CORDE.

The findings of Rommetveit (1968) also fit an input-testing model. Rommetveit found that when SHAR is exposed to one eye along with SHAP to the other eye, the subjects reported SHARP, as if the input passed all the tests for perceiving sHARP. Similarly, Day (1967) found that when RODUCT is presented to one ear, along with PODUCT to the other, the subjects heard pronuct, as if the input passed all the tests for hearing PRODUCT.

In conclusion, further research on input-testing models is recommended to discover the order (if any) in which the tests are usually run; to determine whether different individuals apply different tests to the same input, leading to subject-specific misperceptions (such as Freud's misreading of Hasdrubal as Hamilcar; 1914); and to determine the nature of input testing in other modalities. 


\section{Notes}

This investigation was supported by UCLA Grant 2428 and USPHS Grant 166668-01. The author thanks K. Achevski for running the experiment and analyzing the data, and M. Friedman for lending the experimental equipment. Revision received for publication April 13, 1971.

1. A curious response bias was noted for set words in the set condition. Here the subjects frequently created phonetically compatible misspellings of the set word. For example, they misspelled work as WURK, ADJOURN as ADJURN, and SURVEy as SERVEY. Moreover, these responses were inventions rather than carry-overs from the experimental misspellings WERK, ADJERN, and SURVAY that could have appeared earlier in the session. This response bias suggests that the subjects realized that the stimuli matched the expected word at the phonetic level but were unsure of its orthographic representation.

\section{References}

Corcoran, D. W. J., and Weening, D. L. 1968. Acoustic factors in visual search. Quarterly Journal of Experimental Psychology 20:83-85.

Corcoran, D. W. J. 1966. An acoustic factor in letter cancellation. Nature 210:658.

Corcoran, D. W. J. 1967. Acoustic factors in proof reading. Nature 214:851.

Day, R. 1967. Fusion in dichotic listening. Paper read to the Psychonomic Society, Chicago.

Freud, S. 1914. Psychopathology of everyday life, trans. A. Brill. New York: Penguin.

MacKay, D. G. 1968. Phonetic factors in the perception and recall of spelling errors. Neuropsychologia 6:321-325.

MacKay, D. M. 1967. Ways of looking at perception. In Models for the perception of speech and visual form, ed. W. Wathen-Dunn. Cambridge, Mass.: The M.I.T. Press.

Miller, G. A., Galanter, E., and Pribram, K. D. 1960. Plans and the structure of behavior. New York: Holt.

Rommetveit, R. 1968. Words, meanings, and messages. New York: Academic Press.

Teuber, H. L. 1960. Perception. In Handbook of physiology, ed. John Field, Vol. 3, pp. 1595-1668. Washington, D.C.: American Physiological Society. 